Supplementary Materials for

\title{
Subsurface $\mathrm{pH}$ and carbonate saturation state of aragonite on the
}

\section{Chinese side of the North Yellow Sea: Seasonal variations and controls}

Weidong Zhai ${ }^{1,2}$, Nan Zheng ${ }^{1}$, Cheng Huo ${ }^{1}$, Yi Xu ${ }^{2}$, Huade Zhao ${ }^{1,2}$, Yanwei $\mathrm{Li}^{1}$, Kunpeng Zang ${ }^{1}$, Juying Wang ${ }^{1}$, and Xuemei $\mathrm{Xu}^{1}$

[1] Key Laboratory for Ecological Environment in Coastal Areas (State Oceanic Administration), National Marine Environmental Monitoring Center, Dalian 116023, China

[2] State Key Laboratory of Marine Environmental Science, Xiamen University, Xiamen 361102, China

Correspondence to: W.-D. Zhai (wdzhai@126.com)

The supplementary materials include:

Table S1. Field-measured temperature, salinity, TAlk, DIC, $\mathrm{pH}$ (at $25^{\circ} \mathrm{C}$ ), DO, and calculated $\mathrm{pH}$ (in situ), $\mathrm{fCO}_{2}$ and $\Omega_{\text {arag }}$ in bottom waters.

Figure S1. Distribution of red tides and dominant organism species in Bohai Sea and the North Yellow Sea in 2011. 
Table S1. Field-measured temperature, salinity, TAlk, DIC, $\mathrm{pH}\left(\right.$ at $25^{\circ} \mathrm{C}$ ), DO, and calculated $\mathrm{pH}$ (in situ), fCO and $\Omega_{\text {arag }}$ in bottom waters.

\begin{tabular}{|c|c|c|c|c|c|c|c|c|c|c|c|c|}
\hline Sample\# & $\begin{array}{l}\text { Latitude } \\
\left({ }^{\circ} \mathrm{N}\right)\end{array}$ & $\begin{array}{l}\text { Longitude } \\
\left({ }^{\circ} \mathrm{E}\right)\end{array}$ & $\begin{array}{l}\text { Water depth } \\
\text { (m) }\end{array}$ & $\begin{array}{c}\text { Temperature } \\
\left({ }^{\circ} \mathrm{C}\right)\end{array}$ & Salinity & $\begin{array}{c}\text { TAlk } \\
\left.(\mu \mathrm{mol} \mathrm{kg})^{-1}\right)\end{array}$ & $\begin{array}{c}\text { DIC } \\
\left.(\mu \mathrm{mol} \mathrm{kg})^{-1}\right)\end{array}$ & $\begin{array}{c}\mathrm{pH}_{\mathrm{T}} \\
\left.\text { (at } 25^{\circ} \mathrm{C}\right)\end{array}$ & $\begin{array}{c}\mathrm{DO} \\
\left.(\mu \mathrm{mol} \mathrm{kg})^{-1}\right)\end{array}$ & $\begin{array}{c}\mathrm{pH}_{\mathrm{T}} \\
\text { (in situ) }\end{array}$ & $\begin{array}{c}f \mathrm{CO}_{2} \\
(\mu \mathrm{atm})\end{array}$ & $\Omega_{\text {arag }}$ \\
\hline \multicolumn{13}{|c|}{ Surveying period: $10-17$ May 2011} \\
\hline 1 & 38.7551 & 121.7500 & 55 & 4.32 & 31.78 & 2283 & 2120 & 7.79 & No data & 8.13 & 323 & 1.83 \\
\hline 2 & 38.5841 & 121.2507 & 74 & 5.91 & 31.74 & 2278 & 2097 & 7.86 & No data & 8.15 & 306 & 2.00 \\
\hline 3 & 38.2495 & 121.2497 & 36 & 5.50 & 31.58 & 2312 & 2132 & 7.84 & No data & 8.15 & 310 & 2.02 \\
\hline 4 & 37.9113 & 121.2501 & 20 & 7.46 & 31.54 & 2306 & 2103 & 7.89 & No data & 8.17 & 293 & 2.25 \\
\hline 5 & 37.5837 & 121.7501 & 18 & 10.21 & 31.47 & 2346 & 2140 & 7.88 & No data & 8.13 & 333 & 2.33 \\
\hline 6 & 37.5841 & 122.2480 & 25 & 9.23 & 31.59 & 2314 & 2118 & 7.88 & No data & 8.13 & 331 & 2.21 \\
\hline 7 & 37.5836 & 122.7493 & 30 & 8.01 & 31.57 & 2340 & 2141 & 7.85 & No data & 8.15 & 316 & 2.23 \\
\hline 8 & 37.5833 & 123.2507 & 67 & 6.65 & 32.22 & 2304 & 2134 & 7.80 & No data & 8.10 & 350 & 1.91 \\
\hline 9 & 37.2509 & 123.2491 & 75 & 6.28 & 32.20 & 2298 & 2137 & 7.78 & No data & 8.09 & 361 & 1.83 \\
\hline 10 & 37.2511 & 123.5743 & 73 & 6.60 & 32.31 & 2302 & 2134 & 7.79 & No data & 8.09 & 355 & 1.89 \\
\hline 11 & 37.5841 & 123.5850 & 71 & 6.59 & 32.26 & 2303 & 2135 & 7.79 & No data & 8.10 & 353 & 1.89 \\
\hline 12 & 38.2500 & 123.5843 & 71 & 5.65 & 32.12 & 2307 & 2124 & 7.81 & No data & 8.14 & 311 & 2.01 \\
\hline 13 & 38.2498 & 123.2521 & 63 & 4.83 & 32.12 & 2306 & 2150 & 7.77 & No data & 8.10 & 353 & 1.78 \\
\hline 14 & 38.2509 & 122.7504 & 52 & 5.62 & 32.06 & 2317 & 2162 & 7.73 & No data & 8.08 & 370 & 1.78 \\
\hline 15 & 38.2504 & 122.2508 & 52 & 3.55 & 31.97 & 2321 & 2163 & 7.75 & No data & 8.12 & 332 & 1.79 \\
\hline 16 & 38.2499 & 121.7502 & 50 & 3.99 & 31.90 & 2310 & 2154 & 7.74 & No data & 8.11 & 341 & 1.77 \\
\hline 17 & 38.7500 & 122.2485 & 52 & 3.44 & 32.04 & 2321 & 2176 & 7.69 & No data & 8.09 & 361 & 1.68 \\
\hline 18 & 38.7502 & 122.7512 & 52 & 3.62 & 32.12 & 2322 & 2183 & 7.68 & No data & 8.07 & 381 & 1.62 \\
\hline 19 & 38.7501 & 123.2500 & 56 & 4.50 & 32.05 & 2288 & 2126 & 7.79 & No data & 8.12 & 332 & 1.81 \\
\hline 20 & 38.7494 & 123.5835 & 52 & 6.04 & 32.15 & 2286 & 2125 & 7.82 & No data & 8.09 & 355 & 1.82 \\
\hline 21 & 39.2498 & 123.8278 & 37 & 6.49 & 31.36 & 2255 & 2079 & 7.84 & No data & 8.14 & 315 & 1.97 \\
\hline 22 & 39.6598 & 123.6613 & 13 & 8.39 & 29.38 & 2161 & 2011 & 7.84 & No data & 8.08 & 358 & 1.74 \\
\hline 23 & 39.5330 & 123.4994 & 20 & 5.73 & 30.33 & 2207 & 2028 & 7.88 & No data & 8.17 & 280 & 1.99 \\
\hline
\end{tabular}




\begin{tabular}{|c|c|c|c|c|c|c|c|c|c|c|c|c|}
\hline Sample\# & $\begin{array}{l}\text { Latitude } \\
\left({ }^{\circ} \mathrm{N}\right)\end{array}$ & $\begin{array}{c}\text { Longitude } \\
\left({ }^{\circ} \mathrm{E}\right)\end{array}$ & $\begin{array}{l}\text { Water depth } \\
(\mathrm{m})\end{array}$ & $\begin{array}{c}\text { Temperature } \\
\left({ }^{\circ} \mathrm{C}\right)\end{array}$ & Salinity & $\begin{array}{c}\text { TAlk } \\
\left(\mu \mathrm{mol} \mathrm{kg}{ }^{-1}\right)\end{array}$ & $\begin{array}{c}\text { DIC } \\
\left.(\mu \mathrm{mol} \mathrm{kg})^{-1}\right)\end{array}$ & \begin{tabular}{|c|}
$\mathrm{pH}_{\mathrm{T}}$ \\
(at $\left.25^{\circ} \mathrm{C}\right)$
\end{tabular} & $\begin{array}{c}\text { DO } \\
\left.(\mu \mathrm{mol} \mathrm{kg})^{-1}\right)\end{array}$ & $\begin{array}{c}\mathrm{pH}_{\mathrm{T}} \\
\text { (in situ) }\end{array}$ & $\begin{array}{c}f \mathrm{CO}_{2} \\
(\mu \mathrm{atm})\end{array}$ & $\Omega_{\text {arag }}$ \\
\hline 24 & 39.2495 & 123.4169 & 39 & 5.15 & 31.66 & 2267 & 2105 & 7.83 & No data & 8.12 & 330 & 1.83 \\
\hline 25 & 39.2378 & 123.0003 & 35 & 5.69 & 31.24 & 2249 & 2083 & 7.82 & No data & 8.13 & 321 & 1.88 \\
\hline 26 & 39.0445 & 122.5006 & 35 & 6.61 & 31.29 & 2251 & 2091 & 7.83 & No data & 8.09 & 352 & 1.81 \\
\hline \multicolumn{13}{|c|}{ Surveying period: 22-24 June 2011} \\
\hline 27 & 37.8947 & 123.0598 & 61 & 6.42 & 32.14 & 2297 & 2156 & 7.74 & 273 & 8.03 & 415 & 1.65 \\
\hline 28 & 38.4317 & 123.4783 & 66 & 7.09 & 32.15 & 2299 & 2128 & 7.82 & 301 & 8.10 & 353 & 1.92 \\
\hline 29 & 38.9748 & 123.9076 & 56 & 9.74 & 31.93 & 2281 & 2093 & 7.88 & 306 & 8.10 & 350 & 2.10 \\
\hline 30 & 39.2084 & 124.0863 & 41 & 10.91 & 31.65 & 2271 & 2083 & 7.89 & 303 & 8.09 & 362 & 2.12 \\
\hline 31 & 39.2215 & 123.6356 & 49 & 8.68 & 31.84 & 2281 & 2102 & 7.85 & 297 & 8.10 & 353 & 2.01 \\
\hline 32 & 38.7412 & 123.3595 & 57 & 7.28 & 32.13 & 2295 & 2123 & 7.84 & 305 & 8.10 & 351 & 1.94 \\
\hline 33 & 38.7479 & 122.4999 & 55 & 3.94 & 32.03 & 2304 & 2180 & 7.70 & 289 & 8.02 & 422 & 1.49 \\
\hline 34 & 38.1577 & 122.4834 & 51 & 4.85 & 31.89 & 2298 & 2169 & 7.73 & 278 & 8.03 & 418 & 1.54 \\
\hline 35 & 37.7701 & 122.4809 & 35 & 6.82 & 31.66 & 2305 & 2164 & 7.77 & 274 & 8.03 & 420 & 1.67 \\
\hline 36 & 37.5956 & 122.4742 & 25 & 14.15 & 31.47 & 2342 & 2164 & 7.86 & 224 & 8.01 & 462 & 2.10 \\
\hline 37 & 37.7002 & 121.9915 & 22 & 9.37 & 31.60 & 2322 & 2152 & 7.84 & 289 & 8.06 & 394 & 1.96 \\
\hline 38 & 38.1987 & 121.9979 & 56 & 3.84 & 32.02 & 2298 & 2177 & 7.71 & 285 & 8.02 & 428 & 1.46 \\
\hline 39 & 38.6956 & 122.0063 & 53 & 4.60 & 31.91 & 2298 & 2162 & 7.74 & 294 & 8.05 & 394 & 1.60 \\
\hline 40 & 38.6668 & 121.6252 & 61 & 5.62 & 31.81 & 2287 & 2138 & 7.78 & 292 & 8.07 & 373 & 1.72 \\
\hline 41 & 38.4034 & 121.3270 & 50 & 6.27 & 31.75 & 2278 & 2129 & 7.79 & 291 & 8.06 & 383 & 1.72 \\
\hline 42 & 38.1072 & 121.2174 & 32 & 11.28 & 31.39 & 2309 & 2127 & 7.87 & 281 & 8.07 & 391 & 2.09 \\
\hline 43 & 37.9113 & 121.1586 & 22 & 12.09 & 31.47 & 2315 & 2120 & 7.91 & 300 & 8.08 & 374 & 2.24 \\
\hline \multicolumn{13}{|c|}{ Surveying period: 24-31 July 2011} \\
\hline 44 & 38.7503 & 121.7503 & 53 & 7.37 & 31.89 & No data & 2146 & 7.76 & No data & No data & No data & No data \\
\hline 45 & 38.5826 & 121.2493 & 71 & 6.52 & 31.78 & No data & 2160 & 7.73 & No data & No data & No data & No data \\
\hline 46 & 38.2502 & 121.2502 & 34 & 13.91 & 31.01 & No data & No data & 7.81 & No data & No data & No data & No data \\
\hline 47 & 37.9114 & 121.2510 & 20 & 15.84 & 31.07 & 2305 & 2135 & 7.81 & No data & 7.98 & 501 & 2.04 \\
\hline
\end{tabular}




\begin{tabular}{|c|c|c|c|c|c|c|c|c|c|c|c|c|}
\hline Sample\# & $\begin{array}{l}\text { Latitude } \\
\left({ }^{\circ} \mathrm{N}\right)\end{array}$ & $\begin{array}{c}\text { Longitude } \\
\left({ }^{\circ} \mathrm{E}\right)\end{array}$ & $\begin{array}{l}\text { Water depth } \\
(\mathrm{m})\end{array}$ & $\begin{array}{c}\text { Temperature } \\
\left({ }^{\circ} \mathrm{C}\right)\end{array}$ & Salinity & $\begin{array}{c}\text { TAlk } \\
\left(\mu \mathrm{mol} \mathrm{kg}{ }^{-1}\right)\end{array}$ & $\begin{array}{c}\text { DIC } \\
\left.(\mu \mathrm{mol} \mathrm{kg})^{-1}\right)\end{array}$ & \begin{tabular}{|c|}
$\mathrm{pH}_{\mathrm{T}}$ \\
(at $\left.25^{\circ} \mathrm{C}\right)$
\end{tabular} & $\begin{array}{c}\text { DO } \\
\left.(\mu \mathrm{mol} \mathrm{kg})^{-1}\right)\end{array}$ & $\begin{array}{c}\mathrm{pH}_{\mathrm{T}} \\
\text { (in situ) }\end{array}$ & $\begin{array}{c}\mathrm{fCO}_{2} \\
(\mu \mathrm{atm})\end{array}$ & $\Omega_{\text {arag }}$ \\
\hline 48 & 37.5853 & 121.7508 & 18 & 14.95 & 31.11 & 2294 & 2127 & 7.79 & No data & 7.98 & 489 & 1.99 \\
\hline 49 & 37.5838 & 122.2471 & 24 & 14.85 & 31.16 & 2302 & 2149 & 7.75 & No data & 7.95 & 537 & 1.86 \\
\hline 50 & 37.5844 & 122.7477 & 30 & 12.04 & 31.20 & 2265 & 2117 & 7.76 & No data & 7.98 & 481 & 1.77 \\
\hline 51 & 37.5842 & 123.2493 & 67 & 5.91 & 32.06 & 2301 & 2166 & 7.70 & No data & 8.02 & 426 & 1.59 \\
\hline 52 & 37.2503 & 123.2496 & 71 & 5.66 & 31.92 & 2306 & 2176 & 7.67 & No data & 8.02 & 433 & 1.55 \\
\hline 53 & 37.5828 & 123.5838 & 68 & 6.74 & 32.05 & 2292 & 2159 & 7.73 & No data & 8.01 & 443 & 1.58 \\
\hline 54 & 38.2501 & 123.5833 & 68 & 9.67 & 31.88 & 2287 & 2125 & 7.78 & No data & 8.04 & 412 & 1.85 \\
\hline 55 & 38.2494 & 123.2519 & 63 & 8.89 & 31.99 & 2287 & 2130 & 7.78 & No data & 8.04 & 412 & 1.81 \\
\hline 56 & 38.2492 & 122.7512 & 51 & 5.42 & 32.10 & 2299 & 2170 & 7.71 & No data & 8.02 & 433 & 1.54 \\
\hline 57 & 38.2500 & 122.2579 & 52 & 4.21 & 31.98 & 2299 & 2190 & 7.68 & No data & 7.98 & 470 & 1.37 \\
\hline 58 & 38.2498 & 121.7495 & 48 & 6.07 & 31.81 & 2293 & 2196 & 7.69 & No data & 7.92 & 559 & 1.27 \\
\hline 59 & 38.7494 & 122.2498 & 50 & 6.72 & 32.09 & 2293 & 2162 & 7.76 & No data & 8.00 & 448 & 1.57 \\
\hline 60 & 38.7505 & 122.7488 & 50 & 9.75 & 31.98 & 2288 & 2124 & 7.80 & No data & 8.04 & 407 & 1.89 \\
\hline 61 & 38.7488 & 123.2462 & 55 & 11.17 & 31.90 & 2285 & 2119 & 7.82 & No data & 8.03 & 425 & 1.92 \\
\hline 62 & 38.7495 & 123.5812 & 53 & 11.89 & 31.80 & 2278 & 2115 & 7.84 & No data & 8.01 & 445 & 1.89 \\
\hline 63 & 39.2491 & 123.8265 & 35 & 11.89 & 31.80 & 2273 & 2079 & 7.89 & No data & 8.09 & 365 & 2.19 \\
\hline 64 & 39.6662 & 123.8318 & 17 & 19.03 & 30.45 & 2213 & 2008 & 7.91 & No data & 8.03 & 423 & 2.40 \\
\hline 65 & 39.5339 & 123.4995 & 22 & 17.15 & 31.09 & 2247 & 2060 & 7.88 & No data & 8.00 & 453 & 2.20 \\
\hline 66 & 39.2490 & 123.4172 & 39 & 13.27 & 31.75 & 2278 & 2085 & 7.85 & No data & 8.06 & 391 & 2.19 \\
\hline 67 & 39.0440 & 122.4998 & 35 & 14.37 & 31.29 & 2253 & 2082 & 7.82 & No data & 8.00 & 455 & 1.99 \\
\hline \multicolumn{13}{|c|}{ Surveying period: $20-22$ August 2011} \\
\hline 68 & 38.6591 & 122.0018 & 50 & 9.37 & 31.89 & No data & No data & 7.77 & 245 & No data & No data & No data \\
\hline 69 & 38.1928 & 122.0008 & 50 & 7.46 & 31.84 & 2295 & 2179 & 7.67 & 253 & 7.95 & 513 & 1.45 \\
\hline 70 & 38.1619 & 122.4832 & 47 & 5.08 & 32.02 & 2303 & 2202 & 7.64 & 264 & 7.94 & 520 & 1.31 \\
\hline 71 & 38.7480 & 122.4975 & 51 & 11.94 & 31.83 & 2287 & 2139 & 7.78 & 239 & 7.97 & 495 & 1.76 \\
\hline 72 & 38.7416 & 123.3656 & 56 & 14.64 & 31.18 & 2238 & 2093 & 7.78 & 215 & 7.94 & 532 & 1.75 \\
\hline
\end{tabular}




\begin{tabular}{|c|c|c|c|c|c|c|c|c|c|c|c|c|}
\hline Sample\# & $\begin{array}{l}\text { Latitude } \\
\left({ }^{\circ} \mathrm{N}\right)\end{array}$ & $\begin{array}{c}\text { Longitude } \\
\left({ }^{\circ} \mathrm{E}\right)\end{array}$ & $\begin{array}{l}\text { Water depth } \\
(\mathrm{m})\end{array}$ & $\begin{array}{c}\text { Temperature } \\
\left({ }^{\circ} \mathrm{C}\right)\end{array}$ & Salinity & $\begin{array}{c}\text { TAlk } \\
\left(\mu \mathrm{mol} \mathrm{kg}{ }^{-1}\right)\end{array}$ & $\begin{array}{c}\text { DIC } \\
(\mu \mathrm{mol} \mathrm{kg})\end{array}$ & $\begin{array}{c}\mathrm{pH}_{\mathrm{T}} \\
\text { (at } 25^{\circ} \mathrm{C} \text { ) }\end{array}$ & $\begin{array}{c}\text { DO } \\
\left.(\mu \mathrm{mol} \mathrm{kg})^{-1}\right)\end{array}$ & $\begin{array}{c}\mathrm{pH}_{\mathrm{T}} \\
\text { (in situ) }\end{array}$ & $\begin{array}{c}f \mathrm{CO}_{2} \\
(\mu \mathrm{atm})\end{array}$ & $\Omega_{\text {arag }}$ \\
\hline 73 & 39.2212 & 123.6358 & 49 & 19.10 & 31.04 & 2232 & 2044 & 7.90 & 219 & 7.98 & 482 & 2.22 \\
\hline 74 & 39.2089 & 124.0864 & 41 & 19.57 & 30.52 & 2194 & 2002 & 7.94 & 222 & 7.99 & 460 & 2.26 \\
\hline 75 & 38.9714 & 123.9066 & 55 & 17.88 & 30.31 & 2184 & 2029 & 7.85 & 216 & 7.93 & 537 & 1.87 \\
\hline 76 & 38.4324 & 123.4782 & 65 & 12.11 & 31.68 & 2261 & 2127 & 7.76 & 229 & 7.94 & 534 & 1.62 \\
\hline 77 & 37.8962 & 123.0594 & 62 & 9.61 & 31.89 & 2282 & 2163 & 7.71 & 236 & 7.93 & 546 & 1.48 \\
\hline 78 & 37.7696 & 122.4828 & 35 & 17.44 & 30.87 & 2266 & 2124 & 7.77 & 188 & 7.89 & 618 & 1.78 \\
\hline 79 & 37.5971 & 122.4782 & 24 & 20.58 & 30.83 & 2296 & 2162 & 7.74 & 156 & 7.82 & 755 & 1.75 \\
\hline 80 & 37.7001 & 121.9917 & 20 & 19.43 & 30.86 & 2297 & 2164 & 7.73 & 166 & 7.83 & 726 & 1.73 \\
\hline 81 & 37.9032 & 121.1597 & 21 & 20.06 & 30.80 & 2280 & 2112 & 7.83 & 192 & 7.91 & 587 & 2.06 \\
\hline 82 & 38.1054 & 121.2142 & 29 & 18.55 & 30.83 & 2282 & 2128 & 7.78 & 182 & 7.90 & 604 & 1.91 \\
\hline 83 & 38.4004 & 121.3261 & 50 & 10.46 & 31.78 & 2283 & 2137 & 7.74 & 244 & 7.99 & 469 & 1.73 \\
\hline \multicolumn{13}{|c|}{ Surveying period: $18-23$ October 2011} \\
\hline 84 & 38.7551 & 121.7500 & 56 & 11.15 & 31.62 & 2273 & 2165 & No data & No data & 7.88 & 619 & 1.41 \\
\hline 85 & 38.5841 & 121.2507 & 73 & 12.34 & 31.40 & 2260 & 2150 & 7.66 & No data & 7.87 & 633 & 1.43 \\
\hline 86 & 38.2495 & 121.2497 & 35 & 16.86 & 30.73 & 2229 & 2071 & 7.80 & No data & 7.94 & 527 & 1.91 \\
\hline 87 & 37.9113 & 121.2501 & 20 & 18.00 & 30.50 & 2224 & 2018 & 7.97 & 217 & 8.04 & 406 & 2.39 \\
\hline 88 & 37.5841 & 122.2480 & 23 & 18.37 & 30.56 & 2222 & 2044 & 7.86 & 211 & 7.98 & 487 & 2.12 \\
\hline 89 & 37.5836 & 122.7493 & 32 & 17.74 & 30.73 & 2250 & 2055 & 7.89 & 234 & 8.02 & 442 & 2.28 \\
\hline 90 & 37.5833 & 123.2507 & 65 & 7.79 & 31.97 & 2285 & 2194 & 7.62 & No data & 7.87 & 627 & 1.24 \\
\hline 91 & 37.2509 & 123.2491 & 68 & 7.26 & 32.23 & 2286 & 2206 & 7.59 & 188 & 7.85 & 665 & 1.16 \\
\hline 92 & 37.2511 & 123.5743 & 68 & 7.11 & 32.13 & 2296 & 2199 & 7.61 & 198 & 7.90 & 586 & 1.29 \\
\hline 93 & 37.5841 & 123.5850 & 68 & 7.07 & 32.02 & 2286 & 2208 & 7.63 & No data & 7.84 & 671 & 1.14 \\
\hline 94 & 38.2500 & 123.5843 & 68 & 9.87 & 31.87 & 2278 & 2158 & 7.64 & 181 & 7.93 & 543 & 1.50 \\
\hline 95 & 38.2498 & 123.2521 & 63 & 9.22 & 31.88 & 2286 & No data & 7.60 & 176 & No data & No data & No data \\
\hline 96 & 38.2509 & 122.7504 & 50 & 11.10 & 31.68 & 2279 & 2192 & 7.56 & No data & 7.82 & 729 & 1.24 \\
\hline 97 & 38.2504 & 122.2508 & 50 & 11.91 & 31.52 & 2270 & 2177 & 7.59 & No data & 7.83 & 713 & 1.29 \\
\hline
\end{tabular}




\begin{tabular}{|c|c|c|c|c|c|c|c|c|c|c|c|c|}
\hline Sample\# & $\begin{array}{l}\text { Latitude } \\
\left({ }^{\circ} \mathrm{N}\right)\end{array}$ & $\begin{array}{c}\text { Longitude } \\
\left({ }^{\circ} \mathrm{E}\right)\end{array}$ & $\begin{array}{l}\text { Water depth } \\
\text { (m) }\end{array}$ & $\begin{array}{c}\text { Temperature } \\
\left({ }^{\circ} \mathrm{C}\right)\end{array}$ & Salinity & $\begin{array}{c}\text { TAlk } \\
\left.(\mu \mathrm{mol} \mathrm{kg})^{-1}\right)\end{array}$ & $\begin{array}{c}\text { DIC } \\
\left.(\mu \mathrm{mol} \mathrm{kg})^{-1}\right)\end{array}$ & $\begin{array}{c}\mathrm{pH}_{\mathrm{T}} \\
\left.\text { (at } 25^{\circ} \mathrm{C}\right)\end{array}$ & $\begin{array}{c}\text { DO } \\
\left.(\mu \mathrm{mol} \mathrm{kg})^{-1}\right)\end{array}$ & $\begin{array}{c}\mathrm{pH}_{\mathrm{T}} \\
\text { (in situ) }\end{array}$ & $\begin{array}{c}f \mathrm{CO}_{2} \\
(\mu \mathrm{atm})\end{array}$ & $\Omega_{\text {arag }}$ \\
\hline 98 & 38.2499 & 121.7502 & 49 & 9.15 & 31.77 & 2289 & 2214 & 7.53 & No data & 7.81 & 748 & 1.13 \\
\hline 99 & 38.7500 & 122.2485 & 48 & 11.61 & 31.65 & 2256 & 2167 & 7.61 & 168 & 7.82 & 724 & 1.24 \\
\hline 100 & 38.7502 & 122.7512 & 49 & 11.36 & 31.71 & 2273 & 2156 & 7.59 & 166 & 7.90 & 592 & 1.48 \\
\hline 101 & 38.7501 & 123.2500 & 49 & 12.11 & 31.72 & 2258 & 2153 & 7.63 & 153 & 7.86 & 657 & 1.38 \\
\hline 102 & 38.7494 & 123.5835 & 51 & 13.15 & 31.66 & 2247 & 2144 & 7.66 & 161 & 7.84 & 687 & 1.37 \\
\hline 103 & 39.2498 & 123.8278 & 32 & 16.51 & 30.81 & 2204 & 2039 & 7.81 & No data & 7.97 & 488 & 1.96 \\
\hline 104 & 39.6598 & 123.6613 & 16 & 16.80 & 28.24 & 2076 & 1864 & 7.97 & 237 & 8.13 & 308 & 2.42 \\
\hline 105 & 39.5330 & 123.4994 & 21 & 17.21 & 29.63 & 2159 & 1943 & 7.94 & 230 & 8.10 & 342 & 2.48 \\
\hline 106 & 39.2495 & 123.4169 & 38 & 16.38 & 30.86 & 2209 & 2092 & 7.68 & 157 & 7.85 & 674 & 1.52 \\
\hline 107 & 39.2378 & 123.0003 & 27 & 16.69 & 30.39 & 2187 & 2000 & 7.89 & 239 & 8.03 & 417 & 2.17 \\
\hline 108 & 39.0445 & 122.5006 & 33 & 15.44 & 31.06 & 2221 & 2100 & 7.68 & 138 & 7.87 & 641 & 1.55 \\
\hline \multicolumn{13}{|c|}{ Surveying period: 22-25 November 2011} \\
\hline 109 & 37.4826 & 123.4927 & 73 & 7.82 & 32.25 & 2286 & 2186 & 7.62 & 192 & 7.90 & 587 & 1.30 \\
\hline 110 & 37.6908 & 123.2690 & 68 & 8.04 & 32.13 & 2283 & 2181 & 7.63 & 196 & 7.90 & 581 & 1.32 \\
\hline 111 & 37.8949 & 123.0621 & 61 & 9.01 & 32.03 & 2278 & 2155 & 7.66 & 194 & 7.95 & 519 & 1.50 \\
\hline 112 & 38.1382 & 123.2468 & 64 & 8.65 & 31.93 & 2280 & 2177 & 7.63 & 192 & 7.90 & 590 & 1.33 \\
\hline 113 & 38.4322 & 123.4784 & 65 & 10.45 & 31.85 & 2269 & 2141 & 7.70 & 216 & 7.94 & 524 & 1.56 \\
\hline 114 & 38.7418 & 123.7189 & 58 & 12.09 & 31.69 & 2244 & 2098 & 7.77 & 244 & 7.97 & 487 & 1.72 \\
\hline 115 & 38.9742 & 123.9093 & 56 & 13.19 & 31.42 & 2224 & 2079 & 7.79 & 247 & 7.96 & 499 & 1.73 \\
\hline 116 & 39.2281 & 124.1036 & 31 & 13.67 & 30.39 & 2174 & 2008 & 7.87 & 255 & 8.02 & 418 & 1.92 \\
\hline 117 & 39.2222 & 123.6034 & 40 & 13.43 & 31.29 & 2214 & 2083 & 7.77 & 218 & 7.92 & 551 & 1.61 \\
\hline 118 & 38.7342 & 123.3686 & 58 & 11.33 & 31.78 & 2253 & 2146 & 7.69 & 203 & 7.88 & 622 & 1.39 \\
\hline 119 & 38.7448 & 123.0077 & 54 & 10.36 & 31.84 & 2264 & 2188 & 7.58 & 160 & 7.79 & 766 & 1.13 \\
\hline 120 & 38.7477 & 122.4992 & 54 & 12.02 & 31.63 & 2244 & 2163 & 7.58 & 137 & 7.79 & 776 & 1.18 \\
\hline 121 & 38.1628 & 122.4878 & 50 & 11.93 & 31.55 & 2244 & 2139 & 7.69 & 181 & 7.86 & 641 & 1.38 \\
\hline 122 & 37.5945 & 122.4719 & 26 & 12.76 & 30.81 & 2264 & 2090 & 7.88 & 261 & 8.04 & 413 & 2.03 \\
\hline
\end{tabular}




\begin{tabular}{|c|c|c|c|c|c|c|c|c|c|c|c|c|}
\hline Sample\# & $\begin{array}{l}\text { Latitude } \\
\left({ }^{\circ} \mathrm{N}\right)\end{array}$ & $\begin{array}{c}\text { Longitude } \\
\left({ }^{\circ} \mathrm{E}\right)\end{array}$ & $\begin{array}{l}\text { Water depth } \\
(\mathrm{m})\end{array}$ & $\begin{array}{c}\text { Temperature } \\
\left({ }^{\circ} \mathrm{C}\right)\end{array}$ & Salinity & $\begin{array}{c}\text { TAlk } \\
\left(\mu \mathrm{mol} \mathrm{kg}{ }^{-1}\right)\end{array}$ & $\begin{array}{c}\text { DIC } \\
\left.(\mu \mathrm{mol} \mathrm{kg})^{-1}\right)\end{array}$ & $\begin{array}{c}\mathrm{pH}_{\mathrm{T}} \\
\left.\text { (at } 25^{\circ} \mathrm{C}\right)\end{array}$ & $\begin{array}{c}\mathrm{DO} \\
\left.(\mu \mathrm{mol} \mathrm{kg})^{-1}\right)\end{array}$ & $\begin{array}{c}\mathrm{pH}_{\mathrm{T}} \\
\text { (in situ) }\end{array}$ & $\begin{array}{c}f \mathrm{CO}_{2} \\
(\mu \mathrm{atm})\end{array}$ & $\Omega_{\text {arag }}$ \\
\hline 123 & 37.7842 & 121.9927 & 24 & 12.14 & 30.97 & 2267 & 2102 & 7.84 & 265 & 8.02 & 432 & 1.93 \\
\hline 124 & 38.1991 & 121.9932 & 55 & 12.67 & 31.06 & 2219 & 2062 & 7.85 & 243 & 8.00 & 447 & 1.83 \\
\hline 125 & 38.6675 & 122.0038 & 50 & 12.40 & 31.32 & 2237 & 2131 & 7.70 & 168 & 7.86 & 641 & 1.39 \\
\hline 126 & 38.6675 & 121.6258 & 60 & 12.52 & 31.04 & 2216 & 2075 & 7.79 & 220 & 7.96 & 495 & 1.68 \\
\hline 127 & 38.6351 & 121.4084 & 71 & 12.72 & 30.98 & 2220 & 2064 & 7.81 & 230 & 8.00 & 451 & 1.82 \\
\hline 128 & 38.2660 & 121.2670 & 42 & 12.99 & 30.97 & 2227 & 2070 & 7.83 & 248 & 8.00 & 455 & 1.84 \\
\hline 129 & 37.9105 & 121.1599 & 22 & 12.34 & 30.92 & 2278 & 2103 & 7.85 & 263 & 8.04 & 411 & 2.03 \\
\hline \multicolumn{13}{|c|}{ Surveying period: 09-13 January 2012} \\
\hline 130 & 39.6304 & 123.7999 & 17 & -0.86 & 25.14 & No data & No data & 7.94 & 380 & No data & No data & No data \\
\hline 131 & 39.2501 & 123.8262 & 38 & 5.63 & 31.04 & 2205 & $2067^{\text {a) }}$ & 7.78 & 308 & $8.06^{\mathrm{a})}$ & $374^{\text {a) }}$ & $1.60^{\mathrm{a})}$ \\
\hline 132 & 38.7484 & 123.5749 & 57 & 6.47 & 31.92 & 2243 & $2121^{a)}$ & 7.72 & 301 & $7.99^{a)}$ & $457^{\text {a) }}$ & $1.46^{\mathrm{a})}$ \\
\hline 133 & 37.5771 & 122.2619 & 25 & 3.27 & 31.16 & No data & No data & 7.75 & 331 & No data & No data & No data \\
\hline 134 & 37.5816 & 123.2568 & 63 & 6.74 & 31.98 & 2249 & $2143^{\text {a) }}$ & 7.68 & 286 & $7.94^{a)}$ & $522^{\text {a) }}$ & $1.33^{\mathrm{a})}$ \\
\hline 135 & 38.2555 & 123.5783 & 65 & 6.24 & 32.09 & 2251 & $2148^{\text {a) }}$ & 7.67 & 301 & $7.94^{\mathrm{a})}$ & $521^{\text {a) }}$ & $1.31^{\mathrm{a})}$ \\
\hline 136 & 38.2502 & 122.7508 & 49 & 5.76 & 31.83 & 2243 & $2141^{\text {a) }}$ & 7.67 & 305 & $7.94^{\mathrm{a})}$ & $511^{\text {a) }}$ & $1.30^{\mathrm{a})}$ \\
\hline 137 & 38.7452 & 122.7481 & 47 & 5.14 & 31.60 & 2229 & $2107^{a)}$ & 7.73 & 311 & $8.01^{\mathrm{a})}$ & $423^{\text {a) }}$ & $1.46^{\mathrm{a})}$ \\
\hline \multicolumn{13}{|c|}{ Surveying period: 13-15 May 2012} \\
\hline 138 & 37.3899 & 123.3669 & 72 & 7.51 & 32.52 & 2286 & 2100 & No data & No data & 8.12 & 327 & 2.05 \\
\hline 139 & 37.3895 & 123.1604 & 69 & 7.35 & 32.36 & 2285 & 2108 & No data & No data & 8.10 & 343 & 1.96 \\
\hline 140 & 37.3919 & 122.9346 & 32 & 7.81 & 31.56 & 2300 & 2129 & No data & No data & 8.09 & 360 & 1.95 \\
\hline 141 & 37.8942 & 123.0589 & 61 & 7.02 & 32.24 & 2284 & 2137 & No data & No data & 8.04 & 408 & 1.70 \\
\hline 142 & 38.1356 & 123.2485 & 63 & 5.20 & 31.97 & 2289 & 2155 & No data & No data & 8.03 & 412 & 1.57 \\
\hline 143 & 38.4270 & 123.4757 & 65 & 5.32 & 32.02 & 2285 & 2138 & No data & No data & 8.07 & 377 & 1.69 \\
\hline 144 & 38.7400 & 123.7171 & 58 & 6.32 & 32.10 & 2288 & 2101 & No data & No data & 8.15 & 305 & 2.07 \\
\hline 145 & 38.9722 & 123.9044 & 54 & 6.56 & 32.01 & 2280 & 2079 & No data & No data & 8.18 & 283 & 2.19 \\
\hline 146 & 39.2251 & 124.1026 & 28 & 6.41 & 31.05 & 2237 & 2051 & No data & No data & 8.17 & 289 & 2.06 \\
\hline
\end{tabular}




\begin{tabular}{|c|c|c|c|c|c|c|c|c|c|c|c|c|}
\hline Sample\# & $\begin{array}{l}\text { Latitude } \\
\left({ }^{\circ} \mathrm{N}\right)\end{array}$ & $\begin{array}{c}\text { Longitude } \\
\left({ }^{\circ} \mathrm{E}\right)\end{array}$ & $\begin{array}{l}\text { Water depth } \\
\text { (m) }\end{array}$ & $\begin{array}{c}\text { Temperature } \\
\left({ }^{\circ} \mathrm{C}\right)\end{array}$ & Salinity & $\begin{array}{c}\text { TAlk } \\
\left.(\mu \mathrm{mol} \mathrm{kg})^{-1}\right)\end{array}$ & $\begin{array}{c}\text { DIC } \\
\left.(\mu \mathrm{mol} \mathrm{kg})^{-1}\right)\end{array}$ & $\begin{array}{c}\mathrm{pH}_{\mathrm{T}} \\
\text { (at } 25^{\circ} \mathrm{C} \text { ) }\end{array}$ & $\begin{array}{c}\mathrm{DO} \\
\left.(\mu \mathrm{mol} \mathrm{kg})^{-1}\right)\end{array}$ & $\begin{array}{c}\mathrm{pH}_{\mathrm{T}} \\
\text { (in situ) }\end{array}$ & $\begin{array}{c}f \mathrm{CO}_{2} \\
(\mu \mathrm{atm})\end{array}$ & $\Omega_{\text {arag }}$ \\
\hline 147 & 39.2236 & 123.6062 & 38 & 6.07 & 31.40 & 2256 & 2068 & No data & No data & 8.17 & 288 & 2.07 \\
\hline 148 & 38.7432 & 123.3643 & 57 & 4.97 & 31.91 & 2279 & 2091 & No data & No data & 8.17 & 284 & 2.05 \\
\hline 149 & 38.7463 & 123.0006 & 53 & 3.92 & 31.84 & 2287 & 2137 & No data & No data & 8.10 & 344 & 1.72 \\
\hline 150 & 38.5022 & 122.8788 & 57 & 4.31 & 31.99 & 2290 & 2178 & No data & No data & 7.99 & 462 & 1.38 \\
\hline 151 & 38.2240 & 122.7370 & 53 & 4.49 & 31.77 & 2291 & 2145 & No data & No data & 8.08 & 365 & 1.69 \\
\hline 152 & 38.2240 & 122.7370 & 53 & 4.50 & 31.80 & 2307 & 2143 & No data & No data & 8.12 & 328 & 1.85 \\
\hline 153 & 37.9701 & 122.6129 & 51 & 4.95 & 31.71 & 2291 & 2133 & No data & No data & 8.11 & 342 & 1.80 \\
\hline 154 & 37.6994 & 122.4916 & 29 & 8.75 & 31.13 & 2310 & 2125 & \begin{tabular}{|l|} 
No data \\
\end{tabular} & No data & 8.12 & 340 & 2.11 \\
\hline 155 & 37.7008 & 121.9927 & 22 & 8.64 & 31.15 & 2311 & 2115 & No data & No data & 8.14 & 318 & 2.21 \\
\hline 156 & 38.1993 & 121.9973 & 54 & 4.00 & 31.68 & 2285 & 2132 & No data & No data & 8.11 & 338 & 1.74 \\
\hline 157 & 38.7424 & 122.0050 & 51 & 4.23 & 31.54 & 2264 & 2103 & No data & No data & 8.13 & 320 & 1.80 \\
\hline 158 & 38.6663 & 121.6240 & 61 & 4.39 & 31.40 & 2247 & 2086 & No data & No data & 8.13 & 314 & 1.81 \\
\hline 159 & 38.2665 & 121.2676 & 41 & 5.87 & 31.39 & 2288 & 2138 & No data & No data & 8.07 & 373 & 1.73 \\
\hline 160 & 37.9104 & 121.1583 & 21 & 9.44 & 31.26 & 2299 & 2136 & No data & No data & 8.06 & 400 & 1.90 \\
\hline
\end{tabular}

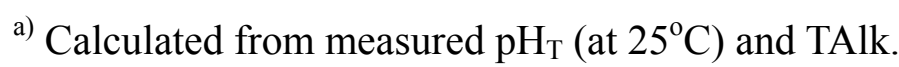




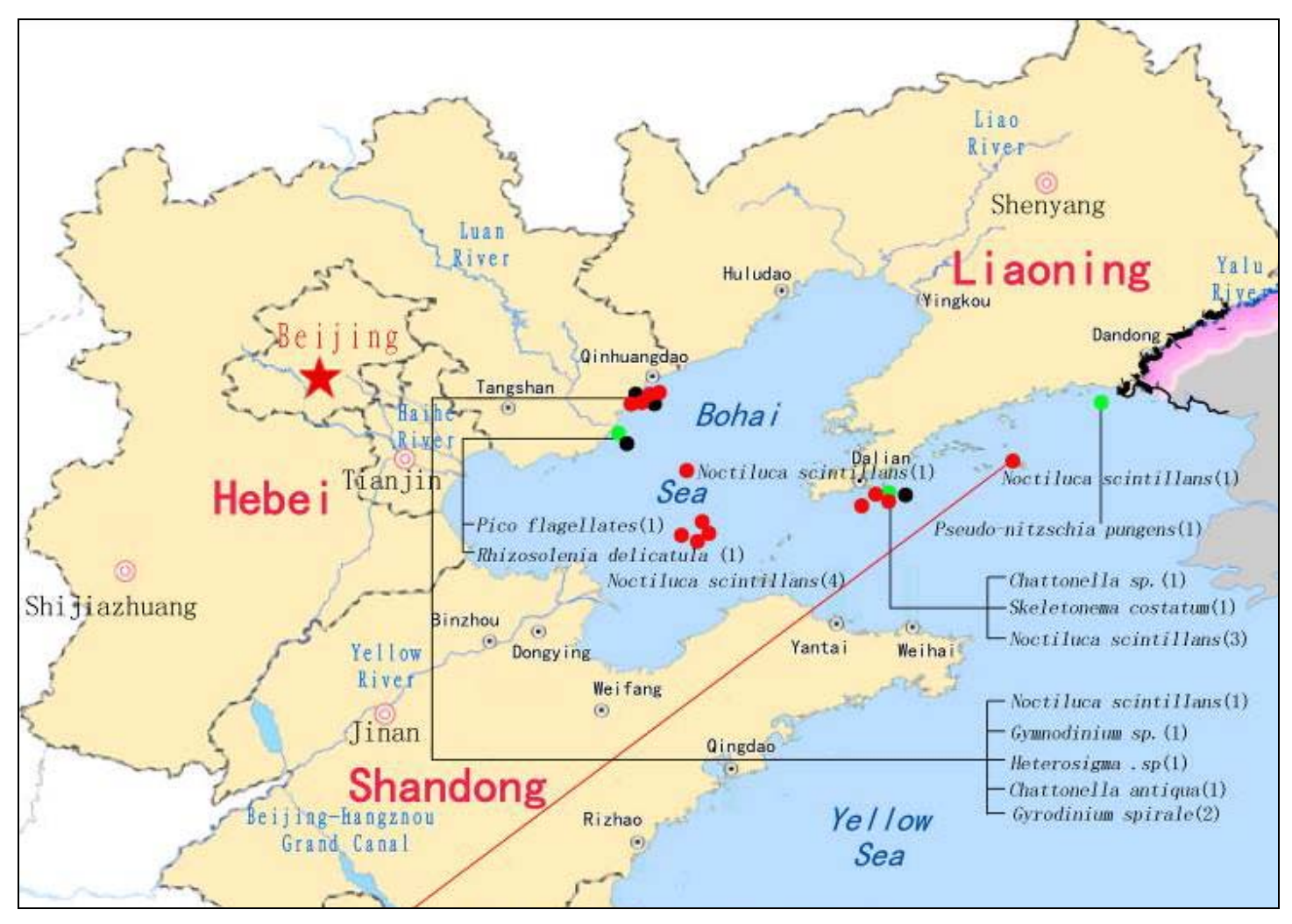

Fig. S1. Distribution of red tides and dominant organism species in Bohai Sea and the North Yellow Sea (NYS) in 2011, based on the Bulletin of Marine Environmental Status of China for 2011, which is released by State Oceanic Administration of China in June 2012. In 2011, seven cases of red tide were recorded in the northern part of the NYS. Among them, four red-tides were caused by Noctiluca scintillans, while the other 3 red tides were induced by Skeletonema costatum, Pseudo-nitzschia pungens, and Chattonella sp. The Chinese version of this figure is available at http://www.soa.gov.cn/zwgk/hygb/zghyhjzlgb/hyhjzlgbml/2011ml/201212/t20121206_21275.html. 